Revista Electrónica Complutense de Investigación en Educación Musical ISSN-e: 1698-7454

http://dx.doi.org/10.5209/RECIEM.57044

\title{
Neoliberalismo, Economía del Conocimiento y Educación Musical ${ }^{1}$
}

\author{
José Luis Aróstegui Plaza²
}

Recibido: 4 de mayo de 2017 / Aceptado: 7 de septiembre de 2017

Resumen. En este artículo se reflexiona sobre la influencia del neoliberalismo y de la economía del conocimiento en la educación en general y en la musical en particular, a fin de tratar el lugar que ocupa y que podría ocupar la música en un currículo entendido como "motor de la economía del conocimiento" actualmente vigente en la mayoría de los países. Para tal fin hago una revisión de la literatura a partir de la cual se tratan diferentes teorías económicas, en particular la del neoliberalismo, también sobre qué se entiende por economía del conocimiento, y de las consecuencias que tienen ambas para la educación y para la educación musical escolar. Al final se concluye que neoliberalismo y economía del conocimiento son dos ámbitos bien distintos, y que mientras la influencia de la primera explica el actual declive de la asignatura, la segunda ofrece un campo de actuación relevante a la música escolar y en realidad a todas las artes y Humanidades, al tiempo que se ofrecen algunas señales a nivel internacional que parecen señalar una mejoría de la música escolar dentro de un currículo dirigido hacia la economía del conocimiento.

Palabras clave: neoliberalismo; economía del conocimiento; sociedad del conocimiento; educación musical escolar; políticas educativas.

\section{[en] Neoliberalism, Knowledge economy and Music Education}

\begin{abstract}
This paper discusses the influence of neoliberalism and the knowledge economy in national curricula, and in school music education in particular, in order to cope with the role school music is playing and it could play in a curriculum conceived "to boost the knowledge economy" currently in effect in most of the countries. For this, I will present some economy theories, with emphasis on neoliberalism, and also what knowledge economy means. Then I will discuss the implications of these economic concepts for both education and school music education and, in the end, I will conclude that neoliberalism and knowledge economy are two different concepts with very different consequences for school music education: while the first brings out a decline of school music education, the latter could give a space for school music and arts and Humanities at large. I will also report some evidences of an improvement of the status of school music education as part of national curriculum supporting the knowledge economy.

Keywords: neoliberalism; knowledge economy; knowledge society; school music education; educational policies.

Sumario: 1. Democracia económica. 2. Qué es la economía del conocimiento. 3. Educación y economía. 3.1. Educación y neoliberalismo. 3.2. Educación y economía del conocimiento. 4. Implicaciones

1 Este artículo es parte del proyecto de investigación I+D EDU2014-58066-P sobre El impacto de la educación musical en la sociedad y en la economía del conocimiento financiado por el Ministerio de Economía y Competitividad del Gobierno de España.

2 Universidad de Granada arostegu@ugr.es
\end{abstract}


para la educación y la educación musical escolar. 4.1. Neoliberalismo y economía del conocimiento, dos realidades diferentes. 4.2. Educación musical escolar y neoliberalismo: mirando al pasado reciente y al presente. 4.3. Educación musical escolar y economía del conocimiento: mirando hacia el futuro. 5. Bibliografia citada.

Cómo citar: Aróstegui Plaza, J.L. (2017). Neoliberalismo, Economía del Conocimiento y Educación Musical, en Revista Electrónica Complutense de Investigación en Educación Musical,14, 11-27.

La mayoría de los sistemas escolares del mundo definen la educación como el "motor de la economía del conocimiento", ya sea a nivel internacional (OCDE, 2010), europeo (Comisión Europea, 2012) o nacional (Jefatura del Estado, 2013). Educar para el ámbito económico no es nuevo; siempre ha sido una de las facetas formativas del sistema educativo, junto con la formación para el ámbito ciudadano (v.g., Pérez Gómez, 2010). Sin embargo, este enfoque de la educación hacia la economía del conocimiento ha supuesto muchos cambios en el currículo y, concretamente para la educación musical escolar en España, el pase de la asignatura de ser obligatoria a optativa tanto en Educación Primaria como en Secundaria, si bien en muchas Comunidades Autónomas se sigue manteniendo como obligatoria. De este y otros cambios cabría concluir que a la administración educativa sólo le importa educar única y exclusivamente para la economía con resultados tangibles a corto plazo, y que para tal fin no se considera útil a la educación musical y artística.

En este artículo me propongo hacer una reflexión sobre la economía del conocimiento aplicada a la educación y sus consecuencias para la educación musical escolar, a fin de tratar si el lugar que ocupa la música y el arte en este nuevo currículo, aún menos relevante de lo que ya lo era previamente, está en coherencia con la aplicación de una educación dirigida a la economía del conocimiento o si por el contrario podría tener un lugar más relevante para tal fin. No trataré, pues, de cuestionar en este artículo si la educación tiene que ser el motor de la economía del conocimiento o no, sino que, partiendo de que el marco legislativo así lo establece, pretendo abordar la cuestión del lugar que ocupa la educación musical escolar y el que podría ocupar dentro de ese tipo de currículo.

Para tal fin hablaré en primer lugar de diferentes teorías económicas, prestando especial atención al neoliberalismo, al ser la teoría económica imperante, para a continuación hablar de la economía del conocimiento. En tercer lugar se tratarán las principales consecuencias de un currículo desarrollado de acuerdo a este enfoque y, por último, se verán las implicaciones que tiene y podría tener todo lo anterior para la educación musical escolar, así como las perspectivas de futuro acerca del lugar que podría ocupar la materia dentro del currículo.

\section{Democracia Económica}

El sistema económico casi único a nivel mundial es el capitalismo. Básicamente se compone de dos elementos: los sistemas de producción, en donde se generan bienes y servicios y que son de propiedad privada en su inmensa mayoría; y el mercado, es decir, la libre circulación de esos bienes, mano de obra incluida, en el que comprador $\mathrm{y}$ vendedor deciden libremente si quieren comprar o vender. Como puede observarse, la producción en principio es totalitaria, pues depende del propietario, mientras que el mercado presupone la libertad de ambas partes. 
Estos rasgos comunes del capitalismo han dado lugar a diferentes teorías económicas que lo justifican y explican, diferenciándose cada una más en el modo y direccionamiento de funcionamiento del capitalismo que en cuestiones de fondo. Las teorías que existen en la actualidad se generan a partir de los años cincuenta del siglo pasado que a su vez parten de otras previas del S. XIX. Así, a partir del liberalismo se generan las teorías económicas de la Escuela de Chicago y de la Escuela de Virginia. La de Chicago se caracteriza por su defensa del mercado y la libre empresa, el rechazo de la intervención del Estado en la economía, y la preferencia por el monetarismo en la política económica, identificándose así con la tendencia liberal más conservadora (González García, 1988). La de Virginia, por su parte, propone la "disminución de los impuestos y control presupuestario mediante la reducción de los servicios sociales y los programas de bienestar social” (ibídem, p. 339).

Como vemos, estas dos teorías tienen en común buscar, por un lado, la desregulación tanto como sea posible del mercado, so pretexto de que se regulará solo por mor de las decisiones tomadas libremente por compradores y vendedores. Por otro, implica un modo de ser del Ser Humano: "la idea del individuo como homo œconomicus, egoísta, racional y maximizador de utilidades" (González García, 1988, p. 336), caracterizado básicamente por ser individual y asocial. En esta línea, Fromm (1964) considera que los rasgos psicológicos compartidos por los miembros de una misma cultura en la sociedad capitalista son: 1) la competencia, principio sobre el que descansa el mercado, y que tiende a aumentar cada vez más con el afán de sobrepasar al competidor; 2) la ganancia por la ganancia, independientemente de su utilidad social y de la satisfacción que genere el proceso de trabajo en sí mismo; 3) la autoridad, es decir, "las relaciones interpersonales en que una persona considera a otra superior a ella misma" (p. 85), que está compuesta de un elemento irracional, al fundamentarse la jerarquía social en la posesión de capital, y de otro racional, al contar con la razón, la moral, y un sentimiento de orgullo y dominio consecuencia del manejo técnico.

Por otro lado, surgen teorías económicas democráticas radicales como reacción a los planteamientos democráticos liberales. Concretamente Keynes incluye mecanismos de corrección de los excesos y desequilibrios del mercado (Morán, 1993), lo que sirve como argumentación para la intervención del Estado en la política económica más allá del arbitraje entre las partes. Downs (1992), por su parte, argumenta que las acciones del gobierno en la economía debe sustentarse en bases tanto económicas como políticas, con lo que igualmente rechaza la autorregulación del mercado. Considera igualmente que gran parte de la teoría económica consiste, en esencia, en probar que los hombres que persiguen sus propios fines pueden, de todos modos, realizar su función social con gran eficiencia, al menos bajo ciertas condiciones, de modo que el homo œconomicus no es algo consustancial al ser humano, sino que la sociedad capitalista lo configura así.

De entre todas estas teorías económicas la que actualmente tiene mayor implantación es el neoliberalismo, basado en las Escuelas de Chicago y Virginia. Se inicia en los Estados Unidos en los años ochenta cuando un grupo de políticos e intelectuales encontraron excesiva la intervención del Estado, considerando que debía hacerse un mayor uso del mercado, constituyendo lo que se llamó el "Consenso de Washington", ya que entre ellos estaban de acuerdo. Consistente en diez principios, tal vez los cinco más importantes sean: 1) el seguimiento estricto de las reglas del mercado; 2) la reducción drástica del gasto público en servicios sociales; 3) la desregulación estatal tanto como sea posible; 4) la privatización; 5) la eliminación de los conceptos 
de "bien público" y "comunidad"3. A partir de ese momento se van generando diversos marcos para desarrollar estos principios que cambiarán el modo de concebir prácticamente todos los ámbitos sociales, también el educativo, tal como se comentará más adelante.

\section{Qué es la Economía del Conocimiento}

A finales del S. XX comienza a surgir un nuevo modo de producir bienes y servicios, basado en la capacidad de generar, acopiar, usar y difundir conocimientos y tecnologías (Ávalos, 1999), cuando hasta ese momento la economía se había basado fundamentalmente en la producción de bienes industriales y de capitales. Así, si observamos la lista de las empresas más importantes de los años 2006 y 2016 (tabla 1), se constata que las empresas que más valían en bolsa hace once años eran una petrolera, una compañía eléctrica, una tecnológica, una gasística, y un banco. En cambio, hace uno, todas estaban directamente relacionadas con las tecnologías digitales:

Tabla 1: Relación de empresas del mundo que más valían en bolsa en el 2006 y el 2016 (fuente: programa La Sexta Columna ${ }^{4}$.

\begin{tabular}{|c|c|}
\hline 2006 & 2016 \\
\hline Exxon & Apple \\
\hline General Electric & Google \\
\hline Microsoft & Microsoft \\
\hline Gazprom & Amazon \\
\hline City Group & Facebook \\
\hline
\end{tabular}

Hablar, por tanto, de economía del conocimiento no es una mera cuestión de nombres, pues aunque el conocimiento siempre ha sido fundamental para el crecimiento económico, ahora se dan una serie de «rupturas» o discontinuidades en la producción económica con respecto a la etapa anterior del capitalismo que suponen un cambio en la producción económica y en el mercado. Dichas rupturas son (David y Foray, 2002):

1. La aceleración en la producción de conocimientos. El ritmo de creación de conocimientos en cualquier rama del saber es exponencial, en un doble sentido: en períodos cada vez más pequeños se producen avances tecnológicos cada vez más importantes; y la información disponible crece también exponencialmente, con el resultado de que el acceso a la información es cada vez más fácil, más cómodo y más barato (Braslavsky, 2006), crecimiento que al mismo tiempo hace que el conocimiento se deprecie, pues resulta válido por un periodo de tiempo menor.

Para más información, véase http://es.wikipedia.org/wiki/Consenso_de_Washington

4 Disponible en http://www.atresplayer.com/television/programas/lasexta-columna/temporada-1/capitulo-167los-nuevos-amos-mundo_2016120200694.html). 
2. La innovación, que se convierte en la actividad dominante y sus fuentes se hacen más difusas. Innovar es fundamental en un contexto tan competitivo como este, más aún con un crecimiento exponencial del conocimiento, por las posibilidades de mejora de cualquier producto o actividad que conlleva, y de que se quede obsoleto el actual si no cambia. Por otro lado, la innovación puede provenir de la investigación y desarrollo, pero cada vez más las ideas nuevas pueden venir de un cliente o de un profano conocedor de la materia, con lo que hay una diversificación, muchas veces inesperada, de la procedencia de la innovación.

3. La revolución de los instrumentos del saber. La entrada en la era digital ha supuesto una revolución en todos los ámbitos de nuestro mundo, y la economía no es una excepción. Las principales repercusiones de las Tecnologías de la Información y la Comunicación en la creación de conocimiento han sido:

a. La creación y puesta a disposición en tiempo real y en cualquier lugar de una cantidad ingente de información.

b. El aumento potencial de las interrelaciones creativas entre, por ejemplo, los creadores del producto, los proveedores y los clientes finales.

c. El tratamiento de la información masiva disponible en las bases de datos (el denominado big data).

d. El desarrollo de sistemas descentralizados y en gran escala de recopilación de datos, de cálculo y de intercambio de los resultados.

El crecimiento del conocimiento, de la innovación y de la digitalización propias de la economía del conocimiento implican que la codificación de la información -es decir, la representación del conocimiento que permite inscribirlo sobre un soporte físico- juega un papel muy importante, pues la transferencia a dicho soporte físico, la búsqueda de los datos deseados y la transmisión de los mismos es imposible sin esa codificación, lo que tiene importantes consecuencias para el modo de aprender del Ser Humano.

Así, en primer lugar, la memoria pasa a tener un lugar secundario, pues toda la información está a un golpe de clic para quienes están inmersos en este mundo digital, por lo que la necesidad, más que en memorizar, está en saber buscar y seleccionar información (v.g., Monereo y Fuentes, 2008). En segundo lugar, implica que, más que aprender el manejo de, por ejemplo, una máquina, necesitamos aprender a aprender, es decir, aprender a adaptarnos a situaciones en cambio constante (v.g., Marcelo, 2001), lo que en ocasiones es casi inmediato (como cuando aprendemos a usar una nueva versión de un sistema operativo) y en otras más complejo (como aprender una nueva técnica quirúrgica o pilotar otro modelo de avión), necesitando entonces un proceso de formación y adaptación. Por último, la codificación, que implica la elaboración de nuevos objetos de conocimiento (p. ej., la lista, la tabla o la fórmula) que facilitan a su vez la creación de otros nuevos (como la taxonomía, la clasificación o la simulación), lo que no es posible ni en una fase de codificación preliteral - gesto y palabra-, o literal -la escritura y el dibujo-, sino cuando estamos en una fase posliteral -el conocimiento está representado por modelos de interacciones estructuradas-.

En suma, "la codificación desempeña una función central en la economía del conocimiento al favorecer los medios de memorización, comunicación y aprendizaje, y constituye asimismo un principio eficaz de creación de nuevos objetos de cono- 
cimiento" (David y Foray, 2002, p. 13) que facilita la creación de comunidades de aprendizaje en las que "la producción y la reproducción de nuevos conocimientos son asumidas por un conjunto no desdeñable de miembros de la comunidad [...]; la comunidad crea un espacio "público" de intercambio de circulación de los saberes y el empleo de nuevas tecnologías de información y comunicación es intenso para codificar y transmitir los conocimientos nuevos" (ibídem, p. 13 y 14), no sin pocas contradicciones y tensiones entre la lógica de la empresa privada, en la que el nuevo conocimiento está controlado de manera exclusiva por cada empresa, y la de esas comunidades de conocimiento cuya esencia reside en la distribución del saber. A pesar de estas contradicciones, las comunidades de aprendizaje son la clave que mantiene la capacidad innovadora de las regiones tecnológicas más dinámicas del mundo (Lam, 2002), como consecuencia de la creación de conocimiento y del aprendizaje colectivo gracias a las redes sociales y profesionales que las conforman.

\section{Educación y Economía}

\subsection{Educación y neoliberalismo}

Lo dicho hasta ahora tiene numerosas implicaciones para el sistema educativo. Con respecto a la aplicación de los principios neoliberales a la educación, ya se mencionó que desde el Consenso de Washington de los ochenta se han venido desarrollando una serie de marcos que lo desarrolla. En lo que a la educación se refiere, el principal acuerdo que la está configurando es el Acuerdo General sobre Aranceles y Servicios (AGCS) de la Organización Mundial de Comercio (OMC), que considera la educación como un servicio más, susceptible, por tanto, de comprarse y venderse en el mercado, antes que como un derecho ciudadano, resultando que "la educación es como un préstamo hipotecario, un servicio de internet o una funeraria; bajo el AGCS, el concepto de educación está profundamente empobrecido y reducido a una sola dimensión" (Dale, 2008, p. 131): la económica.

Esta consideración de la educación como un servicio más no sólo lleva a la aparición de nuevos centros escolares privados necesariamente de élite, al costearse exclusivamente a base del dinero que aportan las familias que pueden permitírselo, sino que supone un riesgo para la escuela pública (Woodford, en prensa, a). Así, en los Estados Unidos, Donald Trump anunció un programa federal de $20.000 \mathrm{mi}$ llones de dólares que gestionarán los Estados de ese país para ayudar "a los niños pobres con resultados académicos bajos a que se matriculen en centros privados" ${ }^{5}$, con lo que se difiere dinero de los centros públicos a los privados. En España se está produciendo esta misma situación, pues el dinero público dedicado a la educación concertada subió un $43 \%$ en una década y ya supera los 5.700 millones de euros anuales ${ }^{6}$, con el resultado de que casi el $80 \%$ de los colegios privados reciben fondos públicos, según los datos del Ministerio de Educación, que indican que

Fuente: Periódico The Washington Post del 8 de septiembre del 2016. Disponible en https://www.washingtonpost.com/news/post-politics/wp/2016/09/08/trump-pitches-20-billion-education-plan-at-ohio-charterschool-that-received-poor-marks-from-state/.

6 Fuente: Periódico Infolibre del 12 de agosto del 2017. Disponible en https://www.infolibre.es/noticias/politica/2017/08/12/el_dinero_publico_dedicado_educacion_concertada_dispara_una_decada_68544_1012.html. 
el nivel de concertación de estudios ha crecido, al tiempo que la escuela pública pierde unidades?

La Organización para la Cooperación y el Desarrollo Económico (OCDE) es la otra gran institución transnacional transfiriendo los principios neoliberales a la educación, concretamente promoviendo la aplicación de criterios empresariales a este ámbito. Hasta tal punto está implicada en esta labor que se ha convertido en un agente fundamental en el diseño de políticas económicas y sociales en todo el mundo y en una "comunidad epistémica" generadora de conocimiento pedagógico (Normand, 2016). No es así de extrañar que la efectividad del sistema educativo sea una de las constantes de estas políticas de reforma educativa no sólo por la estrecha relación entre ambos ámbitos, ítem más cuando, tal como enuncia la Comisión Europea (2012), se concibe la educación como medio para fomentar el crecimiento y la competitividad. Tenemos así una cadena de "recomendaciones" de la Unión Europea, provenientes de la OCDE, que a su vez se reproducen fielmente en la LOMCE y que básicamente se resume en dos ideas: un diseño curricular a partir de competencias y una evaluación de resultados basada en estándares.

En los noventa la OCDE (1996) recuperó y desarrolló este modelo curricular que dio lugar a la Definición y Selección de Competencias (DeSeCo) que a su vez la Unión Europea hizo suyo (Parlamento Europeo, 2006) para luego recomendarlas a los Estados miembros. Se trata, en suma, de un concepto de currículo acorde con los principios económicos que defienden estas instituciones (Martínez Rodríguez, 2012) y que acaban priorizando unas materias, Ciencias, Matemáticas, Tecnología y Lengua, por considerarse que son las que educan para la economía del conocimiento. $\mathrm{Y}$, al priorizar estas materias y sus correspondientes competencias, se hace inevitablemente en detrimento de las restantes.

El currículo basado en competencias proviene del sistema educativo de los años setenta en los Estados Unidos y del desarrollo psicológico correspondiente en el que basar las prácticas de enseñanza consecuentes con tales principios (de la Orden, 2011). En sus orígenes se definió como aquélla basada en el análisis de un perfil futuro o actual que intenta certificar el proceso del estudiante sobre la base de actuaciones demostrables en todos o algunos de los aspectos de dicho perfil (Grant y otros, 1979), lo que supone concebirlas como independientes del contexto en que se desarrollan, es decir, son las mismas para todo el mundo sin importar en dónde se aplican y, en general, hay tendencia a medirlas. Esta necesidad de un currículo independiente del contexto está en coherencia con el principio liberal de que todos somos iguales, de modo que las diferencias que pueda haber en el rendimiento académico son atribuibles al individuo antes que al sistema educativo o a las circunstancias de partida de cada uno; de ahí la importancia de enfatizar la excelencia y el talento de cada persona (OCDE, 2012), de fomentar la competitividad, y de obviar los circunstancias sociales que puedan favorecer o limitar el rendimiento académico.

Al obviar el contexto y recaer la responsabilidad en los individuos, desde esta lógica resulta posible evaluar la calidad de los sistemas educativos nacionales de acuerdo a unos estándares idénticos para todos los países, ya que se persigue la comparación. En estos estándares difícilmente entran la música y las artes que, de hecho, ni se evalúan, centrándose como se centran en tres competencias (la lingüística, la

Fuente: Periódico Público del 16 de diciembre del 2016. Disponible en http://www.publico.es/politica/cuatro-cinco-colegios-privados-reciben.html. 
matemática y la de conocimiento e interacción con el mundo físico) que parecen ser las importantes para estos organismos internacionales y por eso se evalúan, nuevamente en detrimento de las restantes.

\subsection{Educación y Economía del Conocimiento}

De la exposición previa sobre la economía del conocimiento se desprenden muchas consecuencias para el sistema educativo, como el nuevo lugar que debería ocupar la memoria en el proceso de aprendizaje, el empleo de las TIC en el proceso de enseñanza-aprendizaje, la necesidad de saber buscar y seleccionar información en bases de datos, de aprender a aprender a lo largo de toda la vida y poner el énfasis en las competencias generales que permitan adaptarse a los constantes cambios antes que en las específicas que más pronto que tarde quedarán obsoletas, o el trabajar en equipo y con una visión globalizada, antes que compartimentada, del conocimiento. Aun debiendo tener todas ellas consecuencias de calado para la educación escolar, tal vez las más relevantes sean las comunidades de aprendizaje ${ }^{8}$ y el vínculo entre los sistemas de formación y el ámbito laboral.

Comenzando por las comunidades de aprendizaje, son la piedra angular de transformación de la economía, y se caracterizan por sus capacidades múltiples de creación y reproducción de conocimientos, por tener mecanismos de intercambio y de circulación de los conocimientos creados y por la utilización intensiva de las nuevas tecnologías (David y Foray, 2002). Estos modi operandi son transferibles al ámbito educativo, aunque hay algunos inconvenientes para conseguirlo.

Una primera dificultad es que si bien existe una gran aportación de innovaciones educativas, fruto de la experiencia de todos cuantos se esfuerzan por hallar nuevas soluciones a los problemas pedagógicos, la mayor parte de estas innovaciones no se difunden ni intercambian entre los miembros de la comunidad educativa (Hargreaves, 2003).

Otra dificultad estriba en que aquellos sectores económicos que más han crecido dentro de la economía del conocimiento son aquellos en los que la ciencia y la tecnología están estrechamente entrelazados. Sin embargo, en educación, y en realidad en todas las ciencias sociales, la teoría no se relaciona con la práctica como lo hace la física con la ingeniería o la microbiología con la medicina, pues la educación no puede ser entendida unidimensionalmente; aquí la relación teoría-praxis no es lineal, sino dialéctica (Christian, 2001), lo que al mismo tiempo hace que los conocimientos estén poco codificados, al menos en comparación con las ramas científicas. Esta escasa relación entre ciencia y tecnología en educación es lo que hace que las "técnicas" de enseñanza no sean extrapolables de una escuela a otra, dada la importancia del contexto educativo en los resultados del aprendizaje. Puesto que la relación ciencia-tecnología no es la única posible de aplicación ni a la sociedad ni a la economía del conocimiento, David y Foray (2002) sugieren generar modelos que emanen de la práctica como modo de solucionar este problema en educación, invirtiendo así la relación ciencia-tecnología.

Las "comunidades de aprendizaje" como experiencia educativa de éxito basada en la comunicación dialógica (Flecha y Álvarez, 2016) son sobradamente conocidas en educación, sin que tengan necesariamente relación directa con la economía del conocimiento, aunque pueda haber coincidencias. 
Otra dificultad para el desarrollo de estas comunidades de aprendizaje, no sólo en educación, es el de la defensa a ultranza de la propiedad privada propia del neoliberalismo que choca con la libre difusión del conocimiento, que es la esencia de las comunidades de conocimiento, dándose la paradoja de que cuanto más fácil es acceder a la información, más trabas se está poniendo para la libre difusión del conocimiento (David y Foray, 2002). Sin embargo, continuando con el argumento de estos autores, mientras el derecho de propiedad puede tener sentido en productos con acceso más o menos limitado al tener "rivalidad de uso" (p. 21), el conocimiento es ilimitado, y el hecho de que alguien sepa algo no impide que otra persona lo sepa, por lo que carece de sentido dicha rivalidad, antes al contrario: "en este caso, si la creación de derechos de propiedad intelectual excluye a ciertos usuarios potenciales, hay un despilfarro" (ibídem).

La privatización del acceso a la información es una cuestión que claramente afecta al sistema educativo (v.g., Aróstegui, 2011). El uso de licencias DRM protegiendo contenidos multimedia educativos es un ejemplo del sistema escolar, y lo que sucede en enseñanzas universitarias otro incluso más claro; la facilidad con que se accede a la información gracias a las TIC ha ido aumentando a la par que el acceso a ella pagando, bien el usuario final, bien la biblioteca universitaria desde la que se accede. Así mismo, la teoría del aprendizaje móvil (Sharples, 2016) a través de las TIC profundiza en esta cuestión, pues al describir el proceso de aprendizaje como consecuencia de continuas conversaciones en múltiples contextos entre personas mediante tecnologías educativas, suscita de nuevo la cuestión de quién detenta la propiedad del conocimiento y de su desarrollo, lo que entra en conflicto con los derechos de propiedad y de autor, además de con los usos y costumbres habituales del sistema escolar y los límites de acceso al conocimiento que impone el currículo formal, frente a este tipo de aprendizajes difusos y realizados en contextos informales.

La otra consecuencia de la economía del conocimiento para el sistema educativo es la relación que existe entre éste y el ámbito laboral. En esencia, existen dos tipos de sistemas de formación (David y Foray, 2002): uno cerrado y elitista, en el que predomina el conocimiento académico y una distribución de las competencias en dos niveles muy desiguales, y otro abierto que reconoce el valor de la educación académica, pero también el de la formación profesional.

Aunque en principio cabe pensar que el primero pertenece más a un modelo de producción anterior al surgimiento de la economía del conocimiento, en el que el conocimiento teórico se supone superior al práctico al dirigir el primero el trabajo manual del segundo, con la consecuente separación de competencias profesionales que necesitan adquirir unos trabajadores y otros, en la economía del conocimiento acaba derivando en modelos de producción, como el Monotsukuri (Kropp, 2010), en el que los departamentos de diseño trabajan junto con los de producción con el fin de juntar el conocimiento teórico de unos con el práctico de los otros. Los sistemas de formación abiertos, por su parte, también rompen la barrera entre un tipo de formación más académica de otra más manual, con lo que también se ha convertido en un modelo de formación de la economía del conocimiento al facilitar un mercado laboral integrado por diferentes redes sociales y profesionales consecuencia de la interacción entre todas ellas, lo que ha dado lugar a desarrollos tecnológicos y de innovación espectaculares (Lam, 2002); las ya referidas comunidades de aprendizaje.

A pesar de las diferencias evidentes entre los dos sistemas de formación para el ámbito laboral, ambos tienen en común, por un lado, que unen el conocimiento teóri- 
co y positivista con el práctico e interpretativo, siendo precisamente la consideración de ambas la causa de la explosión de la economía del conocimiento. Por otro, ambos sistemas conciben el conocimiento de manera colectiva a partir del saber individual de cada uno de los integrantes, si bien en los sistemas cerrados está limitado al ámbito de la organización, mientras que en el segundo está abierto a toda la comunidad.

\section{Implicaciones para la Educación y la Educación Musical Escolar}

En esta última sección presento algunas ideas a modo de conclusión a las que cabe llegar a partir de la exposición previa sobre la influencia de la economía en la educación y en la educación musical escolar.

\subsection{Neoliberalismo y economía del conocimiento, dos realidades diferentes}

La primera de ellas es que neoliberalismo y economía del conocimiento son dos cosas bien distintas que sin embargo se presentan como si fueran la misma. De la revisión de la literatura se desprende que las críticas que se vierten sobre la educación como motor de la economía del conocimiento son en realidad a la aplicación de los principios neoliberales a la educación, es decir: a concebir los resultados exclusivamente en términos económicos; a considerar la educación como un servicio más; al ataque a la educación pública y la promoción de la privada; a un modelo determinista de evaluación; a ignorar el contexto; a la aplicación de una lógica teoría-praxis lineal; a un modelo de competencias exclusivamente centrado en una formación laboral cortoplacista; $\mathrm{y}$, además, a obviar toda formación humanista y que fomente el pensamiento crítico, así como la ineludible función compensadora de las desigualdades que tiene la educación pública. Ninguna de estas circunstancias tiene necesariamente nada que ver con la economía del conocimiento, ítem más, el propio modelo productivo sufre al neoliberalismo al haber limitaciones a la libre difusión del conocimiento que entra en contradicción con el principio de las comunidades de aprendizaje en el que se sustenta.

Además, la aplicación de los criterios económicos a la educación va más allá de diseñar un currículo y un modelo de evaluación; perfila un tipo de persona, la del homo œconomicus, individualista y asocial ya mencionado, y la escuela no puede ser el agente que forme a este tipo de persona, ni por acción ni por omisión. La necesaria formación para el ámbito laboral que ha de dar la escuela influye además de en los contenidos en las relaciones que se producen en el aula (v.g., Aróstegui, 2000), lo que sin embargo entra en contradicción con la preparación para ejercer la ciudadanía. Y si, como ya se dijo al inicio, el sistema productivo capitalista implica unas relaciones totalitarias, ¿cómo es posible formar simultáneamente para la libertad, la igualdad y la solidaridad propias del ámbito ciudadano? Es imposible asumir estos principios y al mismo tiempo formar a la persona para la competitividad, la ganancia y la autoridad, sea ésta racional o irracional (Fromm, 1964), al menos sin que surjan contradicciones.

Que la escuela prepare simultáneamente para el ámbito ciudadano y laboral supone asignarle una función reproductora no sólo de aquellos conocimientos, creencias y actitudes que conforman el acervo cultural de cada sociedad sino también de las 
contradicciones que surgen como consecuencia de vivir en un sistema político donde todos tenemos en principio los mismos derechos, frente al ámbito económico en el que prima el derecho a la propiedad, a lo que hay que añadir las contradicciones propias de cada uno de los ámbitos económicos, pues mientras la producción es totalitaria, el mercado no. Sin embargo, en las políticas de reforma de un currículo entendido como motor de la economía del conocimiento poco o nada se dice sobre esta formación ciudadana; tampoco se niega y, aunque desde la OCDE se defiendan valores democráticos como los de la justicia social (2011), en la práctica hay una clara diferencia a la hora de aplicar estas ideas frente a los principios individuales de excelencia y talento. La escuela tiene, pues, que formar para el homo sociologicus como metáfora alternativa a la del œconomicus, caracterizado aquél por considerar al Ser Humano como una tabula rasa sobre la que las sociedades y culturas escriben sus valores y propósitos y que, a diferencia del homo œconomicus, no actúa persiguiendo intereses egoístas, sino cumpliendo funciones sociales (Hirsch, Michaels y Friedman, 1990). Así, este individuo sería toda la sociedad en sí mismo y no un individuo aislado.

Por el contrario, incorporar las tecnologías digitales, trabajar en grupo de un modo creativo, compartiendo tareas y conocimiento, adaptarse a los cambios, la codificación y estructuración compleja de la información, vivir en contextos globales y diversos, o aprender en contextos informales y difusos, son características de la economía del conocimiento que la escuela no puede ignorar no sólo porque sean idóneas para ella, sino sobre todo porque dan respuesta a las necesidades sociales además de laborales de nuestro mundo actual. La escuela no puede permanecer ajena a los cambios producidos no sólo en la economía del conocimiento, sino en la sociedad en general y, si el mundo ha cambiado, la escuela también tiene que ir en esta misma línea.

Para ello es necesario fomentar el pensamiento crítico, pero no como lo entiende la OCDE (Winner, Goldstein y Vincent-Lacrin, 2013) cuando lo aplica a la educación artística, es decir, como el desarrollo y aplicación del pensamiento abstracto y de la resolución de problemas al considerarse que es transferible de las artes a las otras asignaturas, sino tal como lo entiende el filósofo John Dewey; como la búsqueda de la verdad y de la justicia social que requiere que los estudiantes "ejerzan una agencia moral y una responsabilidad social cuestionando y desafiando las tradiciones y los mitos sagrados" (Woodford, en prensa b, p. 16).

Necesitamos este tipo de pensamiento crítico para defendernos de los riesgos de la economía del conocimiento neoliberal, en donde el ámbito privado desaparece para convertirse en una fuente de extracción de datos, por el momento en el ámbito virtual, pero que ya ha comenzado en el real ${ }^{9}$, y en el que dejamos de ser no ya ciudadanos, ni tan siquiera consumidores; nos convertimos en el producto. Necesitamos igualmente un verdadero pensamiento crítico para defender la democracia, a la cual se la está vaciando de contenido en todo el mundo como consecuencia de la economía neoliberal (Brown, 2015), no sólo por valorarse cualquier aspecto social desde una perspectiva exclusivamente económica, también por la manipulación a través de la postverdad y de las pretendidas noticias falsas de los medios de comunicación que dicen lo que no gusta al poder, del supremacismo étnico y la xenofobia, de los funda-

Para más información al respecto, véase el artículo de Ben Tarnoff publicado en el periódico The Guardian el 23 de agosto del 2017. Disponible en https://www.theguardian.com/world/2017/aug/23/silicon-valley-big-data-extraction-amazon-whole-foods-facebook. 
mentalismos nacionalistas y religiosos, o de las leyes mordaza que coartan la crítica política y el control a los controladores. La democracia está en peligro, y la escuela tiene un importante papel que jugar, bien por omisión, y por tanto favoreciendo una formación exclusivamente laboral y acrítica, bien tomando partido por los valores democráticos y por la integración y la cohesión social.

Esta distinción entre neoliberalismo y economía del conocimiento es clave para comprender el pobre papel que está jugando la música escolar en un contexto neoliberal y el que podría jugar para la economía del conocimiento, como veremos en los dos siguientes apartados.

\subsection{Educación musical escolar y neoliberalismo: mirando al pasado reciente y al presente}

Si bien existen experiencias emprendedoras que podrían ajustarse a los principios neoliberales en la escuela y que intentan adecuar la educación musical a las nuevas circunstancias (v.g., Bresler, 2011), la realidad es que la mayoría de la literatura que trata esta cuestión, es para denunciar el declive de la educación musical en prácticamente todo el mundo como consecuencia de la implantación de unas políticas educativas de reforma acordes con los planteamientos neoliberales. Así, por ejemplo, Aróstegui (2016) encuentra cuatro causas para ese declive, de las cuales tres son consecuencia de tales políticas neoliberales: el modelo de currículo basado en competencias; una evaluación abrumadoramente cuantitativa; $y$, como consecuencia de lo anterior, menos recursos de tiempo y dinero disponibles para la educación musical $\mathrm{y}$ artística.

Con respecto al currículo por competencias, las críticas han ido evolucionando como igualmente ha sucedido con el concepto de dichas competencias, pues si bien el inicial netamente instructivo se sigue defendiendo por diversos autores (v.g., Vila y Poblete, 2008), también es posible encontrar un enfoque más cognitivo (como el de Garagorri, 2007) y, en educación musical, otro híbrido en el que coexiste lo competencial con lo humanístico (Carrillo, 2012 y 2015) que tiene en cuenta el contexto social. Las críticas, entonces, en realidad vienen por priorizar unas competencias, las de la lengua materna, la matemática y la científica, necesariamente en detrimento de las restantes, y en particular de la competencia cultural y artística, sin duda la menos desarrollada de todas.

Este énfasis de unas competencias sobre otras no sólo aparece en el desarrollo del currículo, sino en su evaluación, estando como está centrada en lengua, matemáticas y ciencias. Pero, aunque se incluyera la competencia cultural y artística en estos exámenes, difícilmente pueden valorar la esencia de las artes; la emoción, la expresión y el desarrollo estético. ¿Existe un procedimiento que mida si se conocen, comprenden, aprecian y valoran críticamente diferentes manifestaciones culturales y artísticas, utilizarlas como fuente de enriquecimiento y disfrute y considerarlas como parte del patrimonio de los pueblos (la definición de la competencia cultural y artística) que sea independiente del contexto de enseñanza-aprendizaje? Es muy fácil determinar la precisión en la afinación de una tercera mayor, por ejemplo, pero las apreciaciones y valoraciones críticas, son otra cosa bien distinta y, a buen seguro, lo que para unos es una buena apreciación no lo sea para otros, y al revés. En estas evaluaciones internacionales hay un énfasis en lo cuantificable y en la independencia 
del contexto que está afectando a todo el currículo, aunque las consecuencias para la educación musical y artística son aún mayores, llegándose incluso a cuestionar su presencia.

En un informe de la OCDE (Winner, Goldstein y Vincent-Lancrin, 2013) se analiza el impacto de la educación artística para el desarrollo de la innovación, entendido como uno de los pilares de este currículo para la economía del conocimiento. La principal conclusión es que si bien se encuentra que las artes contribuyen al desarrollo de las capacidades cognitivas (memoria, inteligencia...), las "evidencias de cualquier tipo de impacto del aprendizaje artístico en la creatividad y en el pensamiento crítico, o en las capacidades conductuales y sociales es, a grandes rasgos, inconcluyente, en parte debido a un insuficiente volumen de investigación experimental al respecto y también en parte a la dificultad de medir estos niveles" (p. 256). Es decir, sólo las herramientas de medición basadas en medidas estandarizadas perfilan estas políticas educativas de reforma que, para colmo, acaban diciendo qué se incluye y qué no dentro del currículo obligatorio, con el resultado de que aquellas competencias que se adaptan mejor a la relación lineal entre teoría y praxis se evalúan y potencian, y las que no, se relegan. Se trata, pues, de un modelo evaluativo de corte cognitivo (Pereyra, Kotthoff y Cowen, 2013) que se extiende linealmente desde lo político hasta lo práctico negando cualquier tipo de complejidad, y por tanto de las Humanidades y de las Artes y en realidad de nuestro mundo global y de la sociedad del conocimiento, que sin embargo permite la "gobernabilidad comparativa" (ibídem) que persigue esta institución transnacional que nadie ha elegido democráticamente para aplicar sus criterios económicos a la educación.

\subsection{Educación musical escolar y economía del conocimiento: mirando hacia el futuro}

En cuanto a las contribuciones de la música escolar a la economía del conocimiento, la principal conclusión que cabría sacar es que "la totalidad de las prácticas escolares tienen que reevaluarse y [que] debemos de deshacernos de muchas de nuestras tradiciones si es que vamos a ser relevantes para la sociedad a la que estamos encomendados a servir" (Jones, 2007, p. 3). Esta misma idea de una redefinición de la educación musical para la sociedad y la economía del conocimiento la defienden otros autores como Aróstegui (2016) o Woodford (en prensa b) quienes sostienen que la idea de la modernidad y el romanticismo de "el arte por amor al arte" que tradicionalmente ha sustentado la educación musical es un argumento muy débil para justificar su permanencia dentro del currículo. Y lo es porque si alguna asignatura debe de formar parte del exiguo tiempo escolar (exiguo al menos para todo lo que se espera de la educación obligatoria) no puede serlo "porque sea muy importante" o porque los clásicos así lo dijeran; aun pudiendo ser razones de peso, cada una de las materias que se incluyan han de dar respuesta a las necesidades de la escuela, y estas hoy por hoy son las de la sociedad y la economía del conocimiento.

En esta línea de desarrollar una educación musical escolar del S. XXI se sitúa el trabajo de diversos autores, entre ellos el del ya citado Jones. Así, propone dos tareas de la educación musical para la economía del conocimiento (2005). Por un lado, la de promocionar comunidades culturales activas, para lo cual: los centros escolares deberían convertirse en centros artísticos fuera del horario escolar; la administra- 
ción debería contratar a músicos y otros artistas para dinamizasen dichos centros artísticos; y, por último, que la música dentro del horario escolar haga de puente para la participación e implicación en estos centros artísticos. Incentivar el arte está relacionado con la economía del conocimiento porque la música es una parte nada despreciable del sistema económico, moviendo en el caso del Reino Unido 410.000 millones de libras esterlinas en el $2016^{10}$ y 5.000 millones de euros en el 2013 en el de España ${ }^{11}$. Por otro lado, la segunda tarea de la música escolar para la economía del conocimiento sería la de desarrollar trabajadores creativos al ser ésta una de las características necesarias para este modelo productivo de la economía a las que en principio el arte tiene mucho que aportar, desarrollando Jones en el artículo (2005) las características que debiera tener un plan de estudios musicales para este propósito. El autor trata igualmente toda una serie de implicaciones que tendría la música escolar para la sociedad del conocimiento en otro trabajo (Jones, 2007) bajo la triple lente de la música, la sociedad y la educación de Small (1989). A todas esas propuestas y las que cabe inferir de lo expuesto en este artículo como posibles aplicaciones de la música escolar para la economía del conocimiento como la codificación, las comunidades de aprendizaje, el uso de las TIC, etcétera, además habría que añadir la relación entre conocimiento interpretativo o práctico y determinista o teórico que se da en la música y que es otra característica importante, como hemos visto, de los sistemas de formación laboral propios de la economía del conocimiento.

Para que todas estas posibilidades de la música escolar para el desarrollo de un currículo dirigido a la economía del conocimiento se conviertan en realidad hace falta una voluntad política por parte de los responsables de las administraciones educativas transnacionales, europeas, nacionales y autonómicas para llevarlo a la práctica, cuestión que, paradójicamente, está empezando a producirse. A pesar del sombrío panorama anteriormente descrito, podemos encontrar en los Estados Unidos las primeras señales de cambio, pues la ley educativa actualmente en vigor, la Every Student Succeeds Act (ESSA) del 2015 le da un espacio a la música para conseguir un programa educativo completo (well-rounded education program), que además contempla la evaluación del proceso educativo en los nuevos estándares revisados (Aróstegui, en prensa), lo que supone no sólo un espacio para la música dentro del currículo, sino una evaluación más acorde con las características de la educación musical escolar. Cabe preguntarse si la nueva administración estadounidense de Donald Trump mantendrá esta Ley, como así parece ser, pues al menos de momento se ha manifestado a favor de ir más allá de "las tres erres"-lectura, escritura y aritmética ${ }^{12}$, como metáfora de las competencias fuertes-, la Historia y las ciencias (Woodford, en prensa a), por lo que tras al menos quince años de una perspectiva muy desoladora para la música, parece haber motivos para el optimismo, por de pronto en ese país. El énfasis en lengua, matemáticas y ciencias, así como las evaluaciones cuantitativas estandarizadas van a seguir existiendo y teniendo mayor peso que las otras materias y métodos de evaluación, pero al menos empieza a haber un espacio real para otros ámbitos del saber y del Ser Humano que sin duda repercutirán también en la eco-

Fuente: UK Music. Disponible en http://www.ukmusic.org/research/measuring-music-2016/.

11 Fuente: diario ABC, publicado el 27 de noviembre del 2013. Disponible en http://www.abc.es/cultura/musica/20131127/abci-ventas-musica-201311271227.html.

12 A estas tres materias se les denomina "las tres erres" por como suenan estas palabras en inglés: Reading, writing, arithmetics. 
nomía del conocimiento. La educación musical y la artística en general podrían ser, así, una metáfora de la defensa de la ineludible función compensadora de la escuela pública y la llave para que otra economía del conocimiento sea posible.

\section{Referencias Bibliográficas}

Aróstegui, J.L. (2000). Democracia y currículum: la participación del alumnado en el aula de música. Tesis doctoral, inédita. Universidad de Granada.

Aróstegui, J. L. (2011). Retos políticos, ciudadanos y educativos del uso de Internet en la escuela. Revista Electrónica Interuniversitaria de Formación del Profesorado, 14(2), 35 48. Disponible en http://www.aufop.com/aufop/revistas/arta/digital/163/1662.

Aróstegui, J. L. (2016). Exploring the global decline of music education. Arts Education Policy Review, 117(2), 96-103. doi: 10.1080/10632913.2015.1007406.

Aróstegui, J. L. (en prensa). Looking to the Future with Optimism: An Interview with Glenn Nierman on occasion of his recent end of his appointment as NAfME President. Arts Education Policy Review, 119(4).

Ávalos, I. (1999). La sociedad del conocimiento. Revista SIC, 617, 295-297.

Braslavsky, C. (2006). Diez factores para una educación de calidad para todos en el siglo XXI. REICE. Revista Iberoamericana sobre Calidad, Eficacia y Cambio en Educación, 4(2e). Disponible en http://www.redalyc.org/html/551/55140206/.

Bresler, L. (2011). Integrating the arts: Educational entrepreneurship in a school setting. Hellenic Journal of Music, Education and Culture, 2, 5-17. Disponible en http://hejmec.eu/ journal/index.php/HeJMEC/article/download/33/26.

Brown, W. (2015). El pueblo sin atributos. Barcelona: Malpaso.

Burton-Jones, A. (2001). Knowledge capitalism: Business, work, and learning in the new economy. Nueva York: OUP.

Carrillo, C. (2012). The qualities of music teachers: Towards a holistic view of the professional profile. Tesis doctoral: Universidad Autónoma de Barcelona. Disponible en http:// www.tdx.cat/bitstream/handle/10803/96532/cca1de1.pdf?sequence=1.

Carrillo, C. (2015). Competencias profesionales del profesorado de música: de los referentes teóricos a la concreción de una propuesta. Revista Internacional de Educación Musical, 3, 11-21. DOI: 10.12967/RIEM-2015-3-p011-021.

Christians, C. G. (2001). The challenge of writing the critical/cultural essay. En Alexander, A. A. y Potter, W. J. (eds.). How to publish your communication research. An insider's guide. Thousand Oaks: Sage.

Comisión Europea. (2012). Rethinking education: Investing in skills for better socio-economic outcomes. Disponible en http://eur-lex.europa.eu/LexUriServ/LexUriServ.do?uri=CO M:2012:0669:FIN:EN:PDF.

Dale, R. (2008). La globalización económica y la educación: relaciones directas, indirectas y colaterales. En J. L. Aróstegui y J. B. Martínez (Ed.). Globalización, postmodernidad y educación: la calidad como coartada neoliberal. Madrid: Akal-UNIA.

David, P. A. y Foray, D. (2002). Fundamentos económicos de la sociedad del conocimiento. Comercio exterior, 52(6), 472-490.

Downs, A. (1992). Teoría económica de la acción política en una democracia. En A. Battle (coord.). Diez textos básicos de ciencia política (pp. 93-111). Madrid: Ariel. 
Flecha, R. y Álvarez, P. (2016). Comunidades de aprendizaje. Padres y Maestros/Journal of Parents and Teachers, 367, 5. El monográfico completo está disponible en http://revistas. upcomillas.es/index.php/padresymaestros/article/download/7114/6947.

Fromm, E. (1964). Psicoanálisis de la sociedad contemporánea. Madrid: Fondo de

Cultura Económica.

Garagorri, X. (2007). Currículo basado en competencias. Aproximación al estado de la cuestión. Aula de Innovación Educativa, 161, 47-55.

González García, J. M. (1988). Crítica de la teoría económica de la democracia. En J. M. González García y F. Quesada (1988). Teorías de la democracia. Barcelona: Anthropos.

Grant, G., Elbow, P., Ewens, T., Gamson, Z., Kohli, W., Newmann, W. Olesen, V. y Riesman, D. (1979). On competence: A critical analysis of competence-based reforms in higher education. San Francisco: Jossey-Bass.

Hargreaves, A. (2003). Teaching in the knowledge society: Education in the age of insecurity. Nueva York: Teachers College Press.

Hirsch, P., Michaels, S. y Friedman, R. (1990). Clean models vs. dirty hands: Why economics is different from sociology. En S. Zukin y P. DiMaggio (Ed.), Structures of capital: The social organization of the economy (pp. 39-56). Cambridge, Nueva York y Melbourne: Cambridge University Press.

Jefatura del Estado (2013). Ley Orgánica 8/2013, de 9 de diciembre, para la mejora de la calidad educativa. Boletín Oficial del Estado, 295, 97858-97921. Disponible en https:// www.boe.es/boe/dias/2013/12/10/pdfs/BOE-A- 2013-12886.pdf.

Jones, P. (2005). Music education and the knowledge economy: Developing creativity, strengthening communities. Arts Education Policy Review, 106(4), 5-12.

Jones, P. (2007). Music education for society's sake: Music education in an era of global neo-imperial/neo- medieval market-driven paradigms and structures. Action, Criticism, and Theory for Music Education, 6(1). Disponible en http://www.maydaygroup.org/ ACT/v6n1/Jones6_1.pdf.

Kopp, R. (2010). Monotsukuri: Valuing hand-craftsmanship in business. Originariamente publicado en Asahi Weekly, la versión en inglés consultada se encuentra disponible en http://www.japanintercultural.com/en/news/default.aspx?newsID=65.

Lam, A. (2002). Los modelos societales alternativos de aprendizaje e innovación en la economía del conocimiento. Revista Internacional de Ciencias Sociales, 171, 1-23. Disponible en https://pure. royalholloway.ac.uk/portal/files/1187073/lam_Alternative_societal_model_Spanish_version.pdf.

Marcelo, C. (2001). Aprender a enseñar para la Sociedad del Conocimiento. Revista Complutense de Educación, 12(2), 531-593.

Martínez Rodríguez, J. B. (2012). Competences: The key to the new curriculum. En J. Paraskeva and J. Torres (Ed.). Globalisms and power: Iberian educational and curriculum policies (pp. 151-162). Nueva York: Peter Lang.

Monereo, C. y Fuentes, M. (2008). La enseñanza y aprendizaje de estrategias de búsqueda y selección de información en entornos virtuales. En C. Coll y C. Monerero (Ed.). Psicología de la educación virtual: aprender y enseñar con las tecnologías de la información y la comunicación (pp. 386-408). Madrid: Morata.

Morán, F. (1993): La deslegitimación relativa de la democracia. Claves de Razón

Práctica, 33, 10-16.

Normand, R. (2016). The changing epistemic governance of European education. The fabrication of the Homo Academicus Europeanus? Dordrecht: Springer.

OCDE (1996). The knowledge-based economy. Disponible en http://www.oecd.org/sti/scitech/ 1913021.pdf. 
OCDE (2010). The OECD innovation strategy: Getting a head start on tomorrow, OECD Publishing. doi:10.1787/9789264083479-en

OCDE (2011). Divided we stand: Why inequality keeps rising. OECD Publishing. doi:10.1787/9789264119536-en

OCDE (2012). Better skills, better jobs, better lives: A strategic approach to skills policies, OECD Publishing. doi:10.1787/9789264177338-en

de la Orden, A. 2011. El problema de las competencias en la educación general. Bordón, 63(1), 47-61.

Martínez Rodríguez, J.B. (2012). Competences: the key to the new curriculum. En J. Paraskeva y J. Torres (Ed.). Globalisms and power: Iberian educational and curriculum policies. Nueva York: Peter Lang.

Parlamento Europeo y Consejo de la Unión Europea (2006). Recommendation of the European Parliament and of the Council of 18 December 2006 on key competences for lifelong learning (2006/962/EC). Official Journal of the European Union, 30.12.2006, L394/310-L394/318. Disponible en http://eur-lex.europa.eu/LexUriServ/Lex UriServ. do?uriDOJ:L:2006:394:0010:0018:en:PDF.

Pereyra, M., Kotthoff, H.-G. y Cowen, R. (2013). PISA a examen: cambiando el conocimiento, cambiando las pruebas y cambiando las escuelas. Introducción al monográfico. Profesorado. Revista de Currículum y Formación del Profesorado, 17(2), 6-14.

Pérez Gómez, Á. I. (2010). Aprender a educar. Nuevos desafíos para la formación de docentes. Revista interuniversitaria de formación del profesorado, 24(2), 37-60.

Sharples, M., Taylor, J. y Vavoula, G. (2016). A theory of learning for the mobile age. En C. Haythornthwaite, R. Andrews, J. Fransman, y E. M. Meyers (Ed.). The Sage Handbook of Elearning Research (pp.221-247). Londres: Sage.

Small, C. (1989). Música, sociedad, educación. Madrid: Morata.

Stiglitz, J. (2012). El precio de la desigualdad. Barcelona-Madrid: Punto de lectura.

Villa, A. y Poblete, M. (dir.) (2008). Aprendizaje basado en competencias: una propuesta para la evaluación de las competencias genéricas. Deusto: Universidad de Deusto.

Winner, E., Goldstein, T. R. y Vincent-Lancrin, S. (2013). Art for art's sake? Overview, OECD Publishing. doi: 10.1787/9789264180789-en

Woodford, P. (en prensa a). On 'The End of History' and The Global Decline of Music Education? En P. Woodford, Music Education in an Age of Virtuality and Post-Truth.

Woodford, P. (en prensa b). It's the economy, stupid. En P. Woodford, Music Education in an Age of Virtuality and Post-Truth. 